\title{
Dynamic RCS Estimation according to Drone Movement Using the MoM and Far-Field Approximation
}

\author{
Dong-Yeob Lee ${ }^{1} \cdot$ Jae-In Lee ${ }^{2} \cdot$ Dong-Wook Seo ${ }^{3,}$
}

\begin{abstract}
Micro-Doppler signatures from the rotating propellers of a drone can be utilized to distinguish the drone from clutter or airborne organisms with similar radar cross section (RCS) levels, such as birds and bats. To obtain the micro-Doppler signatures of a drone, calculation or measurement of the electric field scattered from the rotating propellers is essential. In this paper, using the relative angle concept and far-field approximation, we propose a way to rapidly estimate the dynamic RCS of a drone with several propellers according to its movement. In addition, based on the fact that the shape of the propeller does not change even if it rotates, we construct an impedance matrix only once and apply the matrix to the method of moments instead of the iterative process of calculating the impedance matrix and inverse matrix for each rotation angle of the propeller. Finally, by using the Fourier transform of the results from the proposed method, the rotation frequencies of the propellers according to the movement of the drone can be obtained.
\end{abstract}

Key Words: Drone, Method of Moments (MoM), Micro-Doppler, Radar Cross Section (RCS), Rotation Frequency.

\section{INTRODUCTION}

Recently, the use of drones has been increasing due to their drop in price and miniaturization. At the same time, drones can be abused, leading to issues like privacy violations. Moreover, there is the risk of collision, and they could also be used to transport illegal materials. However, conventional security systems are generally vulnerable to undetected small targets in the air; hence, interest in drone detection technology has increased.

Radar is an appropriate technology for detecting or tracking a target using its radar cross section (RCS). Therefore, early studies on drone detection mainly focused on estimating or measuring the RCS level of drones as well as airborne organisms that have an RCS level similar to that of drones [1]. Despite the ex- istence of many such studies, the RCS level-based method still has difficulties in distinguishing drones from airborne organisms [2]. To solve this problem, methods based on the signature extracted from the RCS of a target rather than the simple RCS level have been studied [3-13]. Inverse synthetic aperture radar (ISAR) images of small drones were investigated in [4]. Twoand three-dimensional ISAR images of drones are also used to classify drones. On the other hand, the micro-Doppler signatures induced by the flapping of various birds' wings have been also investigated [5, 6]. Additionally, in [7-10], the authors showed the difference between the micro-Doppler signatures of flapping bird wings and rotating drone propellers.

To obtain these micro-Doppler signatures for the detection and tracking of a drone, the measurement or estimation of electric

Manuscript received December 31, 2020 ; Revised March 3, 2021 ; Accepted April 8, 2021. (ID No. 20201231-219J)

${ }^{1}$ Department of Radio Communication Engineering, Interdisciplinary Major of Maritime AI Convergence, Korea Maritime and Ocean University, Busan, Korea.

${ }^{2}$ Interdisciplinary Major of Maritime AI Convergence, Korea Maritime and Ocean University, Busan, Korea.

${ }^{3}$ Division of Electronics and Electrical Information Engineering, Interdisciplinary Major of Maritime AI Convergence, Korea Maritime and Ocean University, Busan, Korea.

"Corresponding Author: Dong-Wook Seo (e-mail: dwseo@kmou.ac.kr)

This is an Open-Access article distributed under the terms of the Creative Commons Attribution Non-Commercial License (http://creativecommons.org/licenses/by-nc/4.0) which permits unrestricted non-commercial use, distribution, and reproduction in any medium, provided the original work is properly cited.

(c) Copyright The Korean Institute of Electromagnetic Engineering and Science. 
fields scattered from the drone's propellers with respect to time is essential. Early studies mostly used the measured dynamic RCS - the squared magnitude of the scattered field with respect to time- - because the estimation of the dynamic RCS from rotating propellers requires a large amount of computation time and resources. The measured dynamic RCS is realistic, but it is difficult to measure RCSs according to various drone situations. For this reason, the dynamic RCSs of drones have been generally only measured from hovering drones remaining in the same position. However, in [10], the authors showed that the dynamic RCSs of drones at aspect angles of $0^{\circ}$ and $90^{\circ}$ are considerably different-that is, the dynamic RCS of a drone is greatly affected by the observation angle. However, it is not easy to measure the dynamic RCS of a drone in the air from various angles.

In conventional commercial electromagnetic (EM) tools, the method of moments (MoM) solver generally performs a series of procedures, specifically mesh generation, construction of the impedance and admittance matrices, and calculation of the scattered field and RCS of a target. For the dynamic RCS of the rotating propellers of a drone, it is assumed that the quasi-static mode is established on a frame-by-frame basis with sampling intervals instead of a time-continuous model [11]. Therefore, MoM solvers repeatedly perform identical procedures for each frame. A substantial amount of time is needed to estimate the dynamic RCS of rotating propellers. Even though the propellers rotate, the shape of the propellers does not change; only the incident and scattered angles change. Therefore, using the relative angle between a rotating propeller and the incident field, mesh generation can be done just once along with the construction of the impedance and admittance matrices. Moreover, if the characteristics of the fields scattered from two types of drone propellers-clockwise (CW) and counter-clockwise (CCW) propellers-and far-field approximation are used, the fields scattered from all of the propellers can be synthesized with the scattered field of a single rotating propeller. This can result in a dramatic reduction in the calculation time.

\section{Proposed Method to Estimate Dynamic RCS of}

\section{ROTATING PROPELLERS}

\section{Dynamic RCS of a Single Propeller}

To calculate the electric field scattered from a single rotating propeller, MoM solvers in commercial EM tools perform a series of procedures sequentially, as shown in Fig. 1(a). The most time-consuming components of these MoM procedures are filling the impedance matrix and then converting it into an admittance matrix. It is well known that the impedance matrix in the MoM is constructed from the interaction among the mesh elements of a scatterer. Therefore, if the mesh is maintained while the scatterer is rotated, the impedance matrix does not

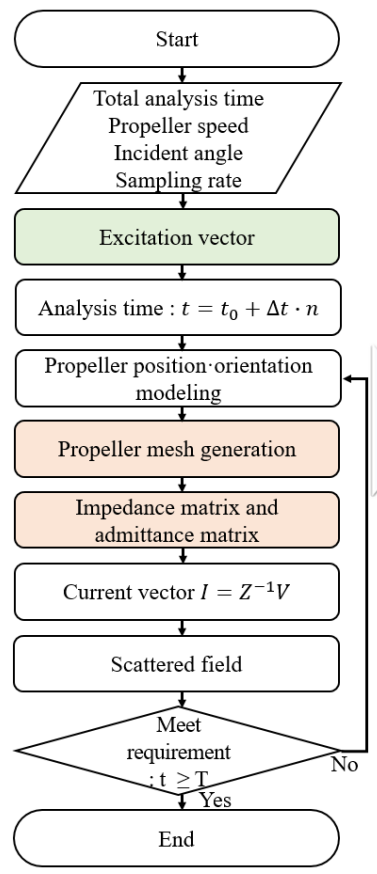

(a)

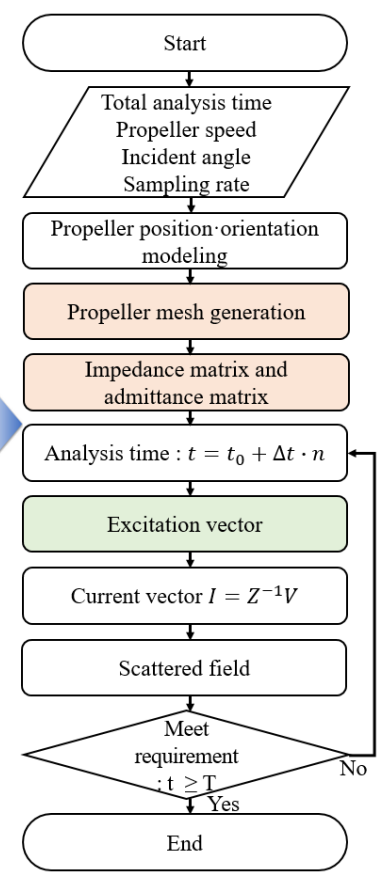

(b)
Fig. 1. Flow chart to estimate RCS of a drone with (a) conventional and (b) proposed methods.

change. That is, the mesh and the impedance matrix generated only once can be used continuously for a rotating propeller. As shown in Fig. 1(b), we reversely rotate the incident angle of the incident field in the rotation direction of the propeller instead of rotating the propeller, and its effect reflects on the excitation vector. Consequently, the current on the elements and the scattered field can obtained.

The electric field scattered from a propeller rotating clockwise, as shown in Fig. 2(a), can be expressed as

$$
E^{s}\left(t ; \theta_{S}, \phi_{S}\right)=S^{\mathrm{CW}}(t) \cdot E^{i}\left(\theta_{\mathrm{inc}}, \phi_{\mathrm{inc}}\right),
$$

where $S^{\mathrm{CW}}(t)$ and $E^{i}$ are the scattering matrix of the CW rotating propeller and the incident electric field, respectively. Instead of rotating the propeller, if the incident azimuth angle, $\phi_{\text {inc }}$, is rotated in the direction opposite to the rotation direction of the propeller, thus keeping the incident elevation angle, $\theta_{\text {inc }}$, fixed,

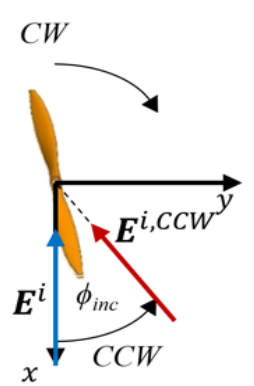

(a)

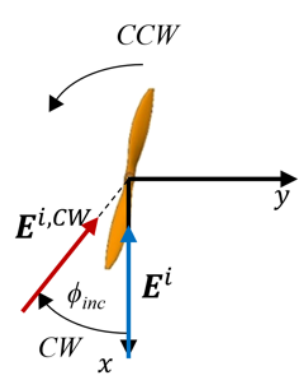

(b)
Fig. 2. Two types of drone propellers: (a) $\mathrm{CW}$ and (b) CCW. 
the same scattered field as Eq. (1) can be obtained by

$$
E^{S}\left(t ; \theta_{S}, \phi_{s}\right)=\mathrm{S}^{C W} \cdot E^{i, C C W}\left(\theta_{\mathrm{inc}}(t), \phi_{\mathrm{inc}}(t)\right) .
$$

On the other hand, drones commonly consist of two types of propellers, namely, mirror-image symmetric $\mathrm{CW}$ and $\mathrm{CCW}$ propellers, as shown in Fig. 2. CW and CCW propellers rotate only clockwise and counter-clockwise, respectively. Because of their mirror-image symmetric characteristics, the co-polarized components of the electric fields scattered from $\mathrm{CW}$ and $\mathrm{CCW}$ propellers are the same, and the cross-polarized components have a phase difference of $180^{\circ}$. Therefore, the scattering matrices of the two types of propellers have the following relationship:

$$
\left[\begin{array}{ll}
S_{\theta \theta}^{C W} & S_{\theta \phi}^{C W} \\
S_{\phi \theta}^{C W} & S_{\phi \phi}^{C W}
\end{array}\right]=\left[\begin{array}{cc}
S_{\theta \theta}^{C C W} & -S_{\theta \phi}^{C C W} \\
-S_{\phi \theta}^{C C W} & S_{\phi \phi}^{C C W}
\end{array}\right] .
$$

From Eq. (3), the electric field scattered from one type of propeller can be easily obtained from the electric field scattered from the other type without additional calculations.

\section{Dynamic RCS of Multiple Propellers}

The geometry of a radar and a drone with 4 propellers is presented in Fig. 3(a). Let us assume that the origin, $O$, of the reference coordinate is the center of the drone, and the nth propeller and the radar are located at $T_{n}$ and $P_{1}$, respectively. If the radar, which faces the head of the drone, is far from the drone, then the backscattered field at the far-field can be approximated as

$$
E_{P_{1}}^{S}(t) \cong \sum_{n=1}^{4} E_{T_{n}}^{S}\left(t ; \theta_{P_{1}}, \phi_{P_{1}}\right) \cdot \exp \left(-j 2 \vec{k} \cdot \vec{r}_{n}\right),
$$

where $E_{T_{n}}^{S}$ is the backscattered field of the $n$th single propeller when the center of the propeller is located at the origin $O$, and $\theta_{P_{1}}$ and $\phi_{P_{1}}$ are the elevation and azimuth angles of the incident field from $P_{1}$, respectively, where $\phi_{P_{1}}=0$ because of the radar located in the heading direction. Here, $\vec{r}_{n}$ is the position vector of the $n$th propeller's center, and $\vec{k}$ is the wave number vector for the operating frequency, $f$, and is defined as $\overline{P_{1} O}$.

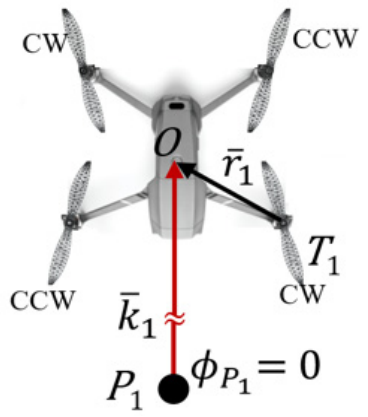

(a)

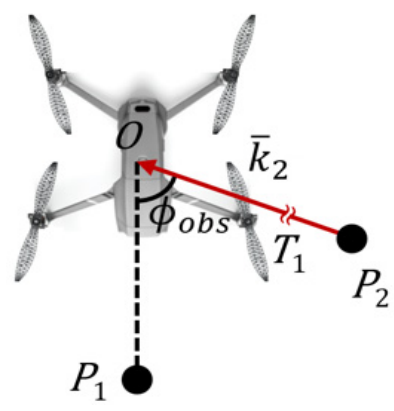

(b)
Fig. 3. Geometry of drone propellers and radar (a) located at $P_{1}$ and (b) $P_{2}$.
Finally, the RCS of the multiple propellers can be estimated by

$$
R C S=\lim _{R \rightarrow \infty} 4 \pi R^{2} \frac{\left|E_{P_{1}}^{s}\right|^{2}}{\left|E^{i}\right|^{2}} .
$$

\section{Dynamic RCS of Multiple Propellers in the Azimuth Plane}

Because a radar can be oriented in any direction, the RCS estimation of multiple propellers for the arbitrary azimuth angle is needed. Let us assume that the observation point is changed from $P_{1}$ to $P_{2}$ by changing only the azimuth angle from $\phi_{P_{1}}$ to $\phi_{P_{2}}$ while maintaining the elevation angle, $\theta_{P_{1}}$. The incident and scattered angles is included in $E_{T_{n}}^{s}\left(t ; \theta_{P_{1}}, \phi_{P_{1}}\right)$ as well as $\vec{k}$ of Eq. (4). If only the wave vector $\vec{k}$ is updated with the moved observation point, the initial direction of the propellers is directed toward $P_{2}$, as shown in Fig. 4(a). This is because the changed angle information is not updated to the scattered field of each propeller, so the initial direction of the propellers is toward the incident wave direction. However, the desired direction of the propellers should be the heading direction as shown in Figs. 3(b) and 4(b). In order to update the changed angles into the scattered field, it is not necessary to recalculate the backscattered field, but only to change the rotation starting angle of $E_{T_{n}}^{S}$ depending on the propeller type, because the backscattered field, $E_{T_{n}}^{S}\left(\theta_{P_{1}}, \phi_{P_{1}}\right)$, was calculated when the single propeller was rotated once. According to the changed azimuth angle, the backscattered fields can be obtained by

$E_{T_{n}}^{s}\left(\theta_{P_{1}}, \phi_{P_{2}}\right)=\left\{\begin{array}{l}E_{T_{n}}^{s}\left(\theta_{P_{1}}, \phi_{P_{1}}+\phi_{\mathrm{obs}}\right), \text { for CW propeller } \\ E_{T_{n}}^{s}\left(\theta_{P_{1}}, \phi_{P_{1}}-\phi_{\mathrm{obs}}\right), \text { for CCW propeller, }\end{array}\right.$

where $\phi_{\mathrm{obs}}$ is the angle between $\overline{O P_{2}}$ and the drone's heading direction. The backscattered field of the multiple propellers for the arbitrary azimuth angle can be synthesized using Eq. (4). As a result, the direction of the radar can be not only at the front but also at the aspect of the drone.

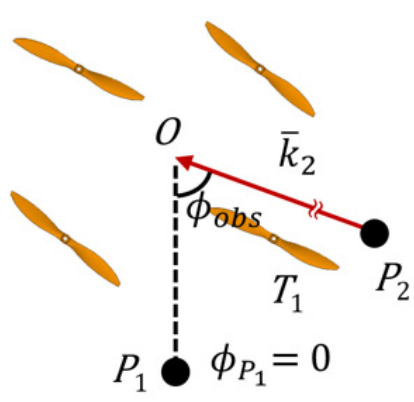

(a)

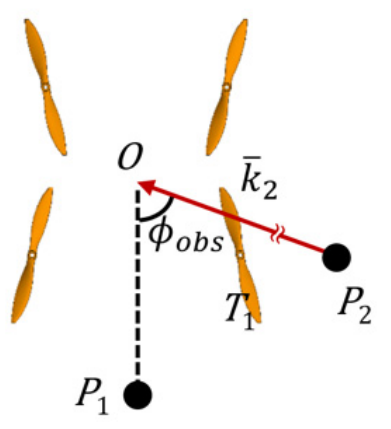

(b)
Fig. 4. Initial directions of propellers due to changed observation point: (a) when the change angle is applied only to the wave vector and (b) when it is applied to both the scattered field and wave vector. 


\section{RESULTS AND DISCUSSION}

The proposed method is implemented based on the MoM with a Rao-Wilton-Glisson (RWG) basis function using the electric field integral equation (EFIE). Since only the impedance matrix for a single propeller of the drone is used in our simulation, the calculation of the admittance matrix, which is an inverse matrix of the impedance matrix, is not time-consuming. Therefore, the admittance matrix was calculated simply using Gauss elimination rather than iterative methods, such as the conjugate gradient algorithm, the biconjugate gradient algorithm, and the generalized minimal residual method.

To verify the proposed method, the monostatic dynamic RCSs of rotating propellers were calculated and compared with the results obtained from a commercial EM tool. First, the radar operating frequency and pulse repetition frequency were set to $9.65 \mathrm{GHz}$ and $20 \mathrm{kHz}$, respectively, in reference to existing radar specifications [12-14]. Next, we choose DJI's Mavic2 [15], which is one of the most popular drones, as the simulation drone model. Since the micro-Doppler is mainly generated by rotating the propellers, we used a computer-aided design (CAD) model of propellers alone without the main body in the simulation. Each propeller was $20.32 \mathrm{~cm}$ long (which results in about $6.5 \lambda$ at $9.65 \mathrm{GHz}$ ) and was made of carbon fiber. Because the dynamic RCSs of propellers with carbon fiber and a perfect electric conductor (PEC) are very similar in terms of RCS level and patterns $[16,17]$, the material of the propellers was simply assumed to be the PEC.

\section{Single Propeller}

The electric fields scattered from a single propeller rotating once a second were calculated at $0.1^{\circ}$ intervals for one second. The backscattered fields with respect to the transmitting and receiving polarizations are shown in Figs. 5 and 6. In the case of co-polarization $(\theta-\theta$ and $\phi-\phi$ polarizations), the backscattered fields of the CW and CCW propellers are identical, while the fields of with cross-polarization $(\theta-\phi$ and $\phi-\theta$ polarizations) are inverse of each other because of their mirrorsymmetric shape and rotation in opposite directions. Their polarimetric characteristics can be used to derive the backscattered field from the other propellers by simply calculating the backscattered field of only one propeller. Therefore, with more propellers, the time for calculating the backscattered field can be reduced.

Fig. 7 shows the dynamic RCSs of the two types of propellers. The proposed method was implemented using MATLAB, and the MoM solver of Altair's FEKO was used as the conventional method. We performed all the simulations using a PC with Intel Core i7-8700 at $3.20 \mathrm{GHz}$ and 64 GB RAM. There was little deviation in the results. As expected, since the proposed

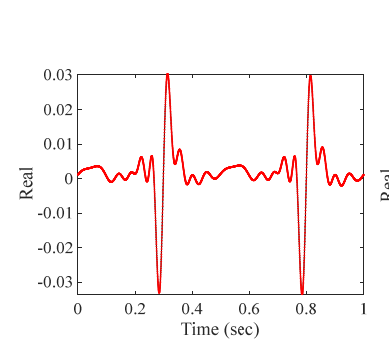

(a)

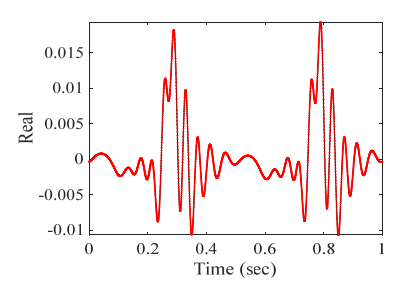

(c)

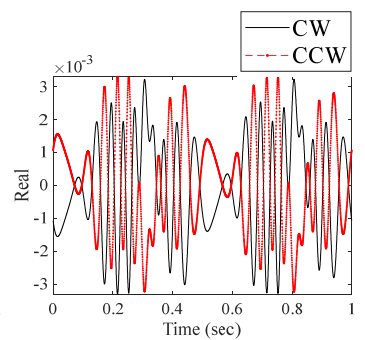

(b)

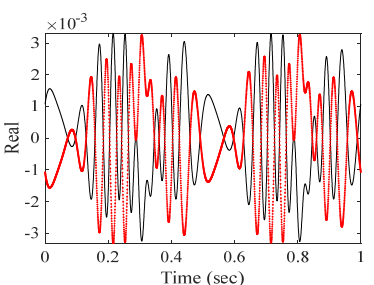

(d)
Fig. 5. Comparison of the real value of the electric field of $\mathrm{CW}$ and CCW propellers. (a) $\theta-\theta$ pol. (b) $\theta-\phi$ pol. (c) $\phi-\phi$ pol. (d) $\phi-\theta$ pol.

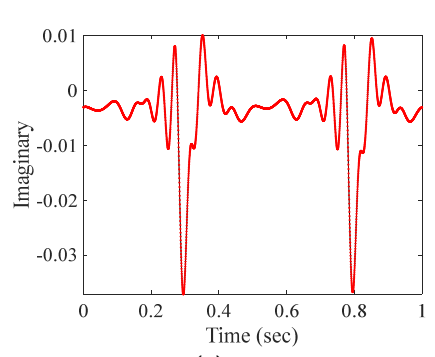

(a)

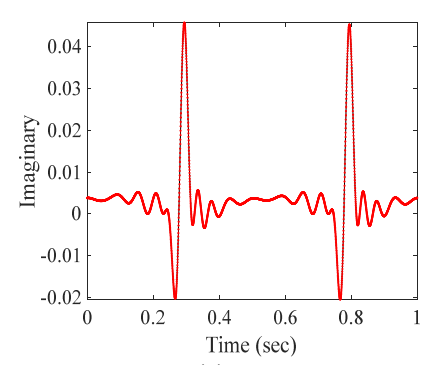

(c)

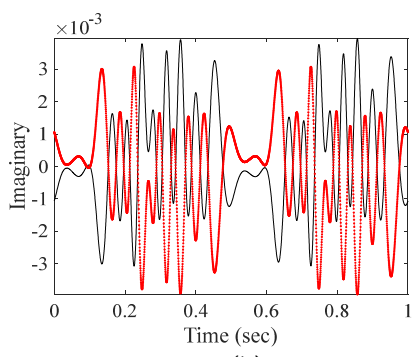

(b)

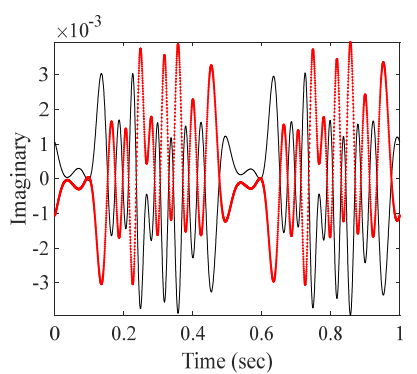

(d)
Fig. 6. Comparison of the imaginary value of the electric field of the $\mathrm{CW}$ and CCW propellers. (a) $\theta-\theta$ pol. (b) $\theta-\phi$ pol. (c) $\phi-\phi$ pol. (d) $\phi-\theta$ pol.

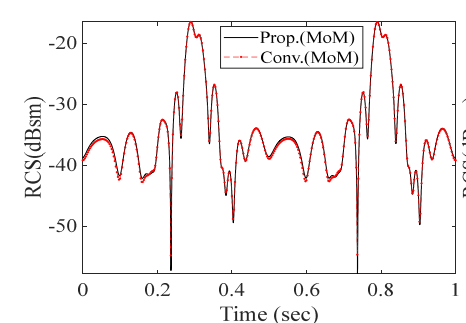

(a)

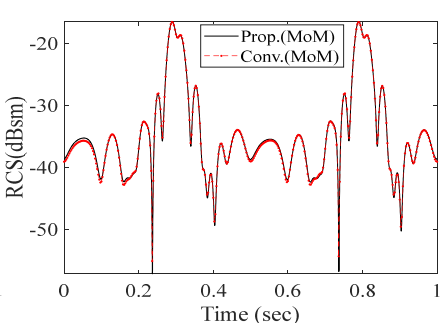

(b)
Fig. 7. Estimated $\mathrm{RCS}_{\theta \theta}$ of single propeller: (a) $\mathrm{CW}$ and (b) $\mathrm{CCW}$ propellers. 
method makes the admittance matrix only one time during the observation time (or dwell time), the proposed method considerably reduces the calculation time of a single propeller in comparison with the conventional method, as summarized in Table 1.

\section{Multiple Propellers of Ascending Drone}

We also simulated a case in which the radar was located in front of a drone with four propellers rotating at $156.6 \mathrm{~Hz}$, as shown in Fig. 3(a), and the elevation and azimuth angles of the incident field were $45^{\circ}$ and $0^{\circ}$, respectively. The dynamic RCSs and backscattered spectrum were calculated during one rotation period. Fig. 8(a) shows the dynamic RCS of the ascending drone. The proposed method was implemented with the procedure shown in Fig. 1(b), while the conventional method, FEKO's MoM solver, was performed at every time step using the procedure shown in Fig. 1(a). As a result, it took a large amount of time to estimate the dynamic RCS of the multiple propellers. Therefore, the conventional method for estimating the dynamic

Table 1. Calculation time for rotating propeller

\begin{tabular}{lc}
\hline & Time (hh:mm:ss) \\
\hline Conventional & $20: 01: 12$ \\
Proposed & $00: 07: 40$ \\
\hline
\end{tabular}

RCS of multiple propellers was implemented using FEKO's multilevel fast multipole method (MLFMM) solver. Fig. 9 shows the frequency response with the application of fast Fourier transform to the dynamic RCS. The frequency of the first peak is $313.2 \mathrm{~Hz}$, which is twice as high as the rotation frequency because the two scattering points at both ends of the propeller rotate.

Fig. 8(b) shows the dynamic RCS when the radar is looking at the aspect of the drone at $\phi_{P_{2}}=70^{\circ}$. Compared to the frontlooking case of Fig. 8(a), the dynamic RCS pattern is considerably changed. However, the back-scattered spectrum is similar. The first peak frequency is also $313.3 \mathrm{~Hz}$, as shown in Fig. 9(b), which means the rotation frequency can be successfully obtained from the frequency-domain of the dynamic RCS.

\section{Multiple Propellers of Advancing Drone}

In considering a drone moving forward with the two front propellers rotating more slowly than the two back propellers, we set the rotation frequencies of the front and back propellers to $125.3 \mathrm{~Hz}$ and $156.6 \mathrm{~Hz}$, respectively. The azimuth and elevation angles between the head of the drone and the radar were $0^{\circ}$ and $45^{\circ}$, respectively. The dynamic RCS of the advancing drone is shown in Fig. 8(c), and the frequencies of the first and second peaks in Fig. 9(c) are $250.6 \mathrm{~Hz}$ and $313.3 \mathrm{~Hz}$, respectively, which are twice as high as the rotation frequencies. However,

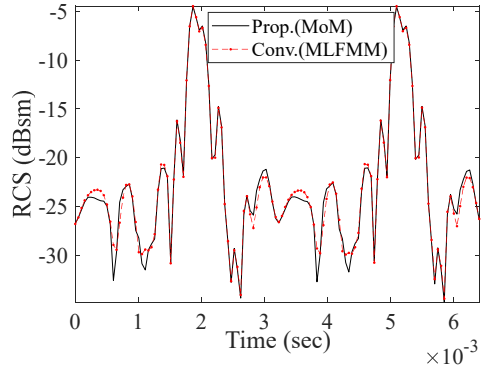

(a)

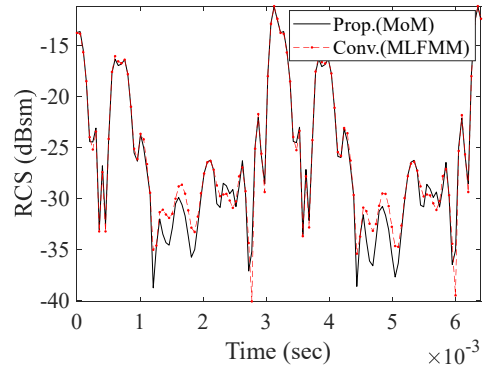

(b)

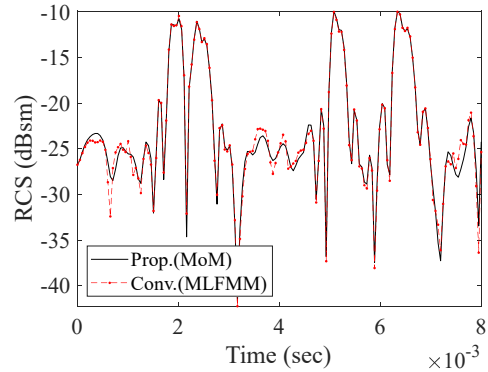

(c)

Fig. 8. Dynamics $\mathrm{RCS}_{\theta \theta}$ calculated by using the proposed and conventional methods when the drone is in (a) ascent, (b) ascent (aspect), and (c) advance.

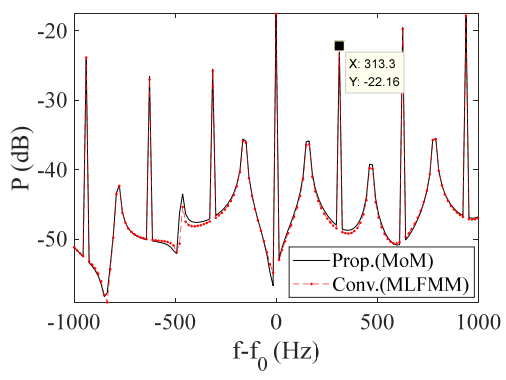

(a)

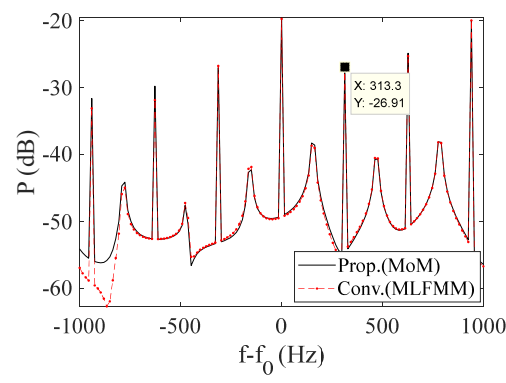

(b)

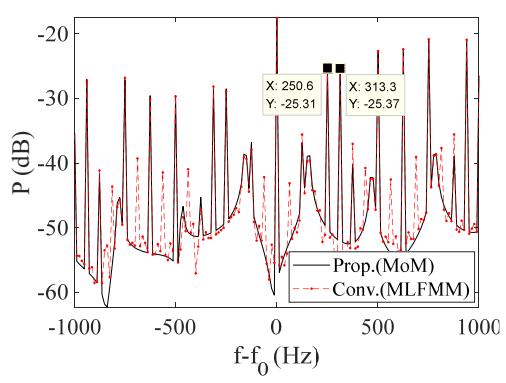

(c)

Fig. 9. Backscattered spectrum calculated by using the proposed and conventional methods when the drone is in (a) ascent, (b) ascent (aspect), and (c) advance. 
there is some discrepancy between the proposed and conventional methods in Fig. 9(c). This discrepancy seems to be due to the assumption that there is no coupling among the propellers for high-speed computation. For the quantitative analysis of the discrepancy, the normalized root-mean-square deviation (NRMSD) between the results of the proposed and conventional methods are summarized in Table 2. The NRMSDs between the results of the proposed method and conventional method are summarized in Table 2. Specifically, the NRMSDs are less than $2 \%$, and the proposed method accurately estimates the dynamic RCSs of the four propellers. Above all, the proposed method considerably reduces the calculation time of the dynamic RCS 8 to 10 times compared with the conventional method, as seen in Table 3.

\section{CONCLUSION}

We introduced a MoM-based method to quickly estimate the dynamic RCSs of rotating objects. Conventional MoM solvers used in commercial EM tools repeatably construct the impedance matrix and its inverse matrix for a rotating object at every time interval, whereas the proposed method constructs the impedance matrix only once by rotating the incident angle instead of rotating the object. This core principle is applicable not only to drones but also to various objects with propellers [18, 19]. In addition, a multi-propeller dynamic RCS is synthesized via far-field approximation assuming that there is no coupling between the propellers and using the characteristics of two mirror-symmetric types of propellers used in drones. The proposed method can rapidly calculate a drone's scattered fields from various angles, especially the aspect. From the estimated dynamic RCS, the rotation frequency of the propeller could be accurately

Table 2. NRMSDs of the difference between the results of the MoM and MLFMM from Fig. 8

\begin{tabular}{lc}
\hline \multicolumn{1}{c}{ Movement } & NRMSD (\%) \\
\hline Ascent & 0.50 \\
Ascent (aspect) & 0.79 \\
Advance & 1.03 \\
\hline
\end{tabular}

Table 3. Calculation time of dynamic RCSs according to the movement of the drone

\begin{tabular}{lcc}
\hline \multirow{2}{*}{ Movement } & \multicolumn{2}{c}{ Time (hh:mm:ss) } \\
\cline { 2 - 3 } & Conventional & Proposed \\
\hline Ascent & $03: 22: 38$ & $00: 23: 25$ \\
Ascent (aspect) & $03: 41: 34$ & $00: 23: 27$ \\
Advance & $04: 19: 17$ & $00: 23: 27$ \\
\hline
\end{tabular}

obtained. Therefore, the proposed method can be effectively used to obtain the micro-Doppler of drones by estimating the dynamic RCS of various drone movements as well as various types of drones, such as drones with four or more propellers and drones with different sizes of propellers, to estimate the dynamic $\mathrm{RCS}$ of drone propellers with various materials in the future.

This work was supported by the Korea Foundation for the Advancement of Science \& Creativity (KOFAC) funded by the Korean Government (MOE) and supported by the Basic Science Research Program through the National Research Foundation of Korea (NRF) funded by the Ministry of Education (No. 2021R1I1A304405).

\section{REFERENCES}

[1] D. Mirkovic, P. M. Stepanian, J. F. Kelly, and P. B. Chilson, "Electromagnetic model reliably predicts radar scattering characteristics of airborne organisms," Scientific Reports, vol. 6, article no. 35637, 2016. https://doi.org/10.1038/srep35637

[2] S. Rahman and D. A. Robertson, "In-flight RCS measurements of drones and birds at K-band and W-band," IET Radar, Sonar \& Navigation, vol. 13, no. 2, pp. 300-309, 2019.

[3] J. S. Patel, F. Fioranelli, and D. Anderson, "Review of radar classification and RCS characterisation techniques for small UAVs or drones," IET Radar, Sonar \& Navigation, vol. 12, no. 9, pp. 911-919, 2018.

[4] M. Pieraccini, L. Miccinesi, and N. Rojhani, "RCS measurements and ISAR images of small UAVs," IEEE Aerospace and Electronic Systems Magazine, vol. 32, no. 9, pp. 28-32, 2017.

[5] B. Torvik, K. E. Olsen, and H. D. Griffiths, "X-band measurements of radar signatures of large sea birds," in Proceedings of 2014 International Radar Conference, Lille, France, 2014, pp. 1-6.

[6] M. Ritchie, F. Fioranelli, and H. Griffiths, "Monostatic and bistatic radar measurements of birds and micro-drone," in Proceedings of 2016 IEEE Radar Conference (RadarConf), Philadelphia, PA, 2016, pp. 1-5.

[7] T. Li, B. Wen, Y. Tian, Z. Li, and S. Wang, "Numerical simulation and experimental analysis of small drone rotor blade polarimetry based on RCS and micro-Doppler signature," IEEE Antennas and Wireless Propagation Letters, vol. 18, no. 1, pp. 187-191, 2019.

[8] M. A. Govoni, "Micro-Doppler signal decomposition of small commercial drones," in Proceedings of 2017 IEEE Radar Conference (RadarConf), Seattle, WA, 2017, pp. 425-429.

[9] S. Rahman and D. A. Robertson, "Radar micro-Doppler signatures of drones and birds at K-band and W-band," 
Scientific Reports, vol. 8, article no. 17396, 2018. https://doi. org/10.1038/s41598-018-35880-9

[10] B. K. Kim, H. S. Kang, and S. O. Park, "Experimental analysis of small drone polarimetry based on micro-Doppler signature," IEEE Geoscience and Remote Sensing Letters, vol. 14, no. 10, pp. 1670-1674, 2017.

[11] P. Pouliguen, L. Lucas, F. Muller, S. Quete, and C. Terret, "Calculation and analysis of electromagnetic scattering by helicopter rotating blades," IEEE Transactions on Antennas and Propagation, vol. 50, no. 10, pp. 1396-1408, 2002.

[12] V. K. Kothapudi and V. Kumar, "Compact $1 \times 2$ and $2 \times 2$ dual polarized series-fed antenna array for X-band airborne synthetic aperture radar applications," Journal of Electromagnetic Engineering and Science, vol. 18, no. 2, pp. 117-128, 2018.

[13] L. Lehmann and J. Dall, "Simulation-based approach to classification of airborne drones," in Proceedings of 2020 IEEE Radar Conference (RadarConf20), Florence, Italy, 2020, pp. 1-6.

[14] P.J. Speirs, A. Schroder, M. Renker, P. Wellig, and A. Murk, "Comparisons between simulated and measured X-band signatures of quad-, hexa-and octocopters," in Proceedings of 2018 15th European Radar Conference (EuRAD), Madrid,

\section{Dong-Yeob Lee}

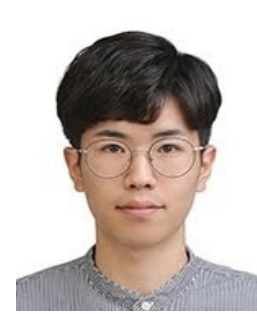

received a B.S degree in radio communication engineering from Korea Maritime and Ocean University, Busan, South Korea in 2021. He is currently working toward his M.S. degree at the same university. His research interests include numerical techniques in areas of the electromagnetics and radar crosssection analysis.

Jae-In Lee

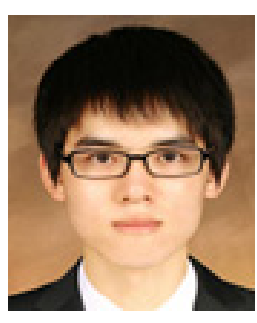

received a B.S. degree in electrical engineering from Kyungpook National University, Daegu, South Korea, in 2012 and Ph.D. degrees in electrical engineering from Korea Advanced Institute of Science and Technology (KAIST), Daejeon, South Korea, in 2019. $\mathrm{He}$ is currently a post-doctoral researcher associate with the Interdisciplinary Major of Maritime AI Convergence, Korea Maritime and Ocean University (KMOU), Busan, South Korea. His research interests include numerical techniques in the areas of electromagnetics, radar cross-section analysis, and inverse synthetic aperture radar imaging.
Spain, 2018, pp. 325-328.

[15] Specification for the DJI Mavic-2 [Online]. Available: https://www.dji.com/kr/mavic-2/info\#specs.

[16] A. Schroder, M. Renker, U. Aulenbacher, A. Murk, U. Boniger, R. Oechslin, and P. Wellig, "Numerical and experimental radar cross section analysis of the quadrocopter DJI Phantom 2," in Proceedings of 2015 IEEE Radar Conference, Johannesburg, South Africa, 2015, pp. 463-468.

[17] M. Ritchie, F. Fioranelli, H. Griffiths, and B. Torvik, "Micro-drone RCS analysis," in Proceedings of 2015 IEEE Radar Conference, Johannesburg, South Africa, 2015, pp. 452456.

[18] S. H. Choi, D. W. Seo, and N. H. Myung, "Scattering analysis of open-ended cavity with inner object," Journal of Electromagnetic Waves and Applications, vol. 21, no. 12, pp. 1689-1702, 2007.

[19] W. Y. Yang, D. J. Yun, and D. W. Seo, "Novel automatic algorithm for estimating the jet engine blade number of insufficient JEM signals," Journal of Electromagnetic Engineering and Science, to be published.

[20] S. N. Makarov. Antenna and EM Modeling with MATLAB. New York, NY: Wiley-Interscience, 2002.

\section{Dong-Wook Seo}

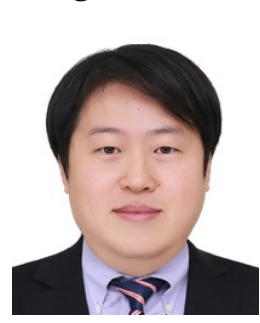

received the B.S. degree in electrical engineering from Kyungpook National University, Daegu, South Korea, in 2003, and M.S. and Ph.D. degrees in electrical engineering from Korea Advanced Institute of Science and Technology (KAIST), Daejeon, South Korea, in 2005 and 2011, respectively. He was a senior researcher for the Defense Agency for Technology and Quality (DTaQ), Daegu, from 2011 to 2012. From 2012 to 2017, he was a senior researcher with the Electronics and Telecommunications Research Institute (ETRI), Daegu. Since September 2017, he has been a faculty member of the Department of Radio Communication Engineering, Korea Maritime and Ocean University (KMOU), Busan, South Korea, where he is currently an associate professor. His current research interests include numerical techniques in the areas of electromagnetics, radar cross-section analysis, wireless power transfer, and radar systems. 